\title{
IRRADIATION-DRIVEN MASS TRANSFER IN LOW-MASS X-RAY BINARIES
}

\author{
PHILIPP PODSIADLOWSKI \\ Institute of Astronomy, Cambridge CB3 OHA, U.K.
}

\begin{abstract}
External irradiation can completely alter the internal structure of the secondary in a low-mass X-ray binary (LMXB). For radiation fluxes as are typically found in LMXBs, the secondary expands by a factor of 2 to 3 in order to relax towards a new state of thermal equilibrium. This relaxation process provides a new mass-transfer driving mechanism for LMXBs, which can drastically alter the evolution of these systems.
\end{abstract}

\section{Stellar Response to External Irradiation}

The main effect of external irradiation is to change the star's surface boundary condition. Thus, if a star has a deep convective envelope, external irradiation can drastically alter the star's internal structure, since the surface boundary condition determines the adiabat of the whole convective layer. The most important physical consequence of external irradiation is to change the degree of ionization of hydrogen, which affects the specific entropy near the surface.

As a result of external irradiation, a star has to adjust to its new surface boundary condition, i.e., relax towards a new equilibrium state (shown in figure 1). This relaxation process (see figure 2) takes place on the thermal timescale of the convective envelope $\left(\sim 10^{7} \mathrm{yr}\right.$ for $\left.M \simeq 0.8 M_{\odot}\right)$. It is worth emphasizing that the energy required to inflate the star to its new equilibrium radius is not supplied by the external radiation flux (i.e., the star is not heated from the outside), but is almost entirely provided by energy generated in the secondary's core. The external radiation almost acts like a catalyst, only changing the surface boundary condition and establishing an isothermal layer below the photosphere, which insulates the interior of the star during the relaxation phase.

The details of the relaxation process are not very sensitive to how deep below the photosphere the external radiation is deposited and what the detailed spectrum of the radiation is (see figure 2). The only necessary requirement is that the energy is deposited near or below the photosphere (possible radiation sources include $\mathrm{X}$ - and $\gamma$-rays above $2 \mathrm{keV}$, near UV below $10 \mathrm{eV}$, secondary synchroton and inverse Compton radiation from the interaction with a relativistic pulsar wind).

\section{Application to LMXBs}

In the following, we discuss - qualitatively - how external irradiation affects the evolution of LMXBs. Generally, we can distinguish four phases: a turn-on phase, a relaxation phase, a quasiequilibrium phase and a turn-off phase. 


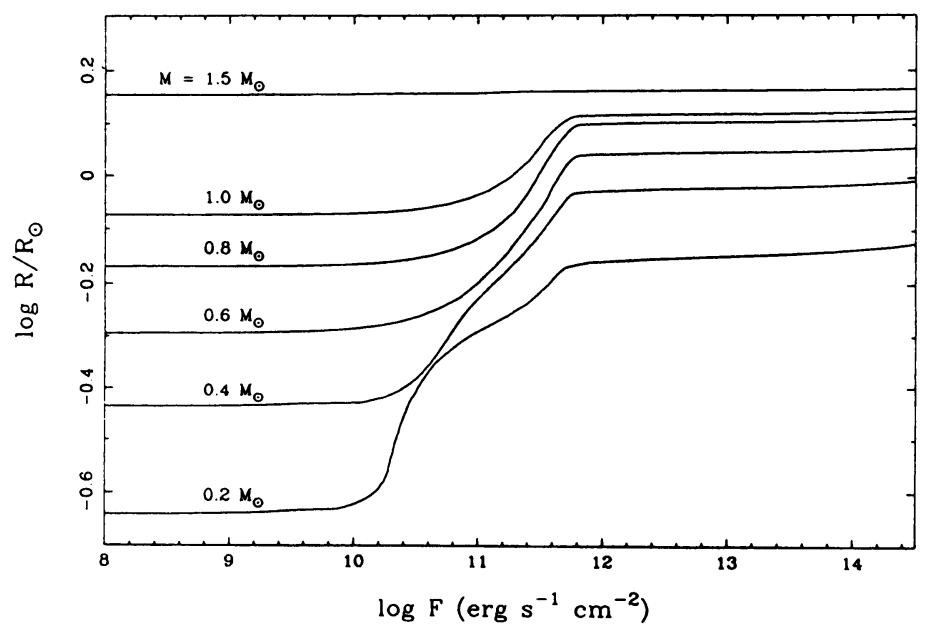

Figure 1. The equilibrium radius, $R$ of irradiated stars as a function of external irradiation flux, $F$, for unevolved stars of mass, $M$, as indicated. Note that there are two flux regimes, separated by a characteristic flux, $F_{\mathrm{ch}} \simeq 10^{11}-10^{12} \mathrm{erg} \mathrm{s} \mathrm{sm}^{-1} \mathrm{~cm}^{-2}$, within which the equilibrium radius, and thus the structure of the star, changes only slowly with external flux. The difference between the regimes is that, for $F \ll F_{\text {ch }}$, hydrogen at the bottom of the irradiated layer is mainly non-ionized, while for $F \gg F_{\mathrm{ch}}$, it is completely ionized (and the star is completely radiative). The fluxes typically found in LMXBs are near the upper end of the shown flux range.

\subsection{THE TURN-ON PHASE}

The turn-on phase is characterized by a strong positive feedback between the mass-transfer rate and the external irradiation flux. As a result, the mass-transfer rate rapidly reaches its steadystate value. (The details of this phase are very sensitive to when significant mass transfer [and the concomitant X-ray flux] starts; in particular, whether the expansion of the secondary starts when it fills its Roche lobe or slightly earlier.)

\subsection{THE "EQUILIBRATION" PHASE $\left(\sim 10^{7} \mathrm{yr}\right)$}

In the equilibration phase, mass transfer is driven by the thermal relaxation of the secondary's envelope. Since the secondary expands during this phase, the orbital period generally also increases.

\subsection{THE QUASI-EQUILIBRIUM PHASE}

After the secondary has achieved its new larger (quasi-)equilibrium state, mass transfer would stop, if there were no other mass-transfer driving mechanism. In practice, gravitational radiation and/or magnetic braking may now become important and determine the further evolution (note, however, that the orbits are larger than in cases without internal irradiation, since the secondary passes through a different sequence of quasi-equilibrium models).

\subsection{THE TURN-OFF PHASE}

Unlike the case without external irradiation, mass transfer stops abruptly, when the external irradiation flux falls below a certain critical flux, $F_{\mathrm{cr}} \sim F_{\mathrm{ch}}$, when, due to recombination of hydrogen in the irradiated layer, the secondary starts to shrink more rapidly than the orbit can shrink due to angular-momentum losses from the system and the system becomes detached. 


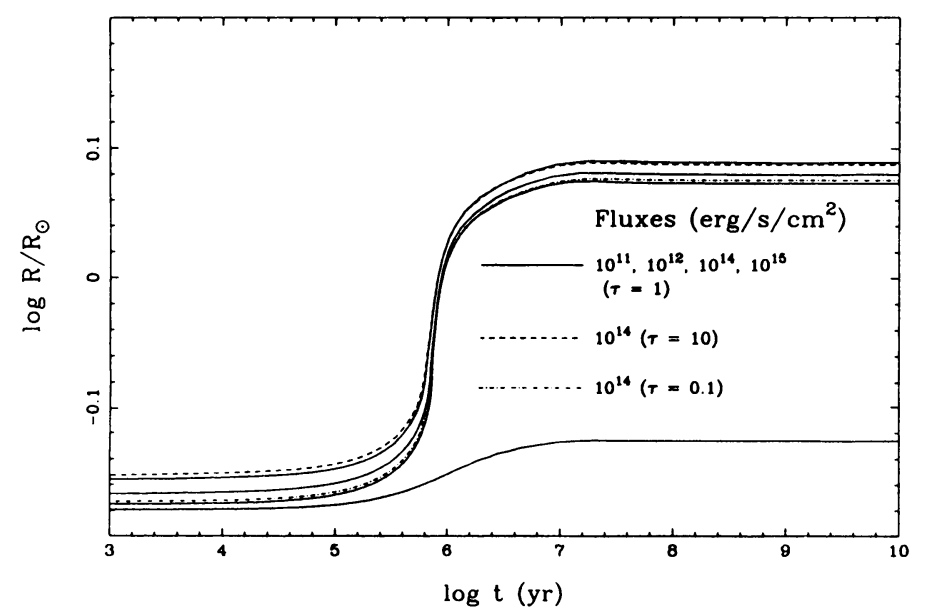

Figure 2. The evolution of the radius during the relaxation phase for a star of $M=0.8 M_{\odot}$ as a function of time for various external fluxes and different penetration depths of the radiation ( $\tau$ denotes the characteristic Thompson optical depth at which the external radiation is deposited). Note the strong resemblance of the individual results.

The details of the evolution of the LMXB phase are very sensitive to the evolutionary state of the secondary and the details of the a angular-momentum loss mechanisms, and not all of these phases may be realized for any particular set of assumptions. Figure 3 illustrates two representative cases.

\section{Implications for LMXBs}

Our calculations suggest that external irradiation effects can explain a variety of observed features of LMXBs that can not easily be understood within the existing theoretical framework.

- If the secondary is a main-sequence star, there is a phase, in which the secondary expands and the orbital period increases. Such period increases are observed at about the predicted rate in several LMXBs (e.g., Cyg X-3 and X 1822-371). Thus, these systems may provide direct observational evidence for the existence of a relaxation phase.

- The radius of the secondary and the orbital period of systems with main-sequence secondaries are significantly larger than in calculations that do not include irradiation effects. As a result, systems like AC $211(4 \mathrm{U} 2127+11)$ can have main-sequence instead of subgiant secondaries.

- In our calculations, mass transfer stops abruptly, when the external flux drops below a critical value. This introduces a low-luminosity cutoff in the luminosity distribution of LMXBs (at $\sim 10^{36} \mathrm{erg} \mathrm{s}^{-1}$ in our model calculation), which is consistent with the observed luminosity distribution.

- When the binary becomes detached, the radius of the secondary is larger by a factor of $2-3$ than its equilibrium radius without external illumination. This may explain why, in systems like the binary millisecond pulsar PSR $1957+20$, the secondary significantly underfills its Roche lobe. Indeed, in our model calculation with a main-sequence secondary, the system at the end of the mass-transfer phase is very similar to PSR $1957+20$.

- LMXBs with subgiant secondaries (with periods less than $\sim 30 \mathrm{~d}$ ) are expected to radiate near the neutron star's Eddington limit (which may explain the high luminosity of systems like Sco X-1), 


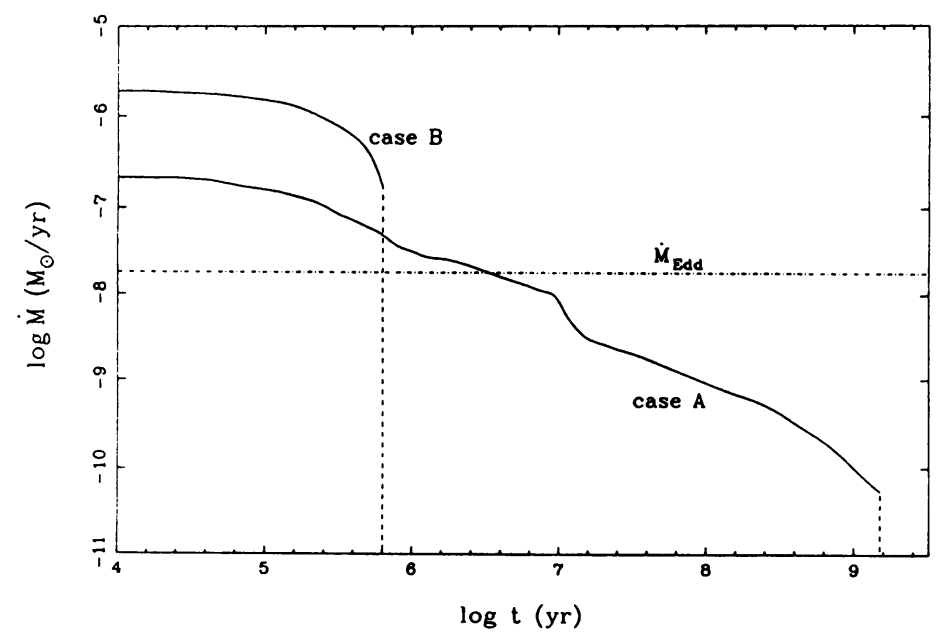

Figure 3. Mass loss rate, $\dot{M}$, of the secondary in an irradiated LMXB as a function of time, $t$, after the beginning of the mass-transfer phase for two illustrative binary cases. In the first case (case A), the secondary is an unevolved star of mass $0.8 M_{\odot}$, in the second case (case B), a slightly evolved subgiant of the same mass. The initial orbital periods are $5.7 \mathrm{hr}$ and $45 \mathrm{hr}$, respectively, and the final periods are $8 \mathrm{hr}$ and $90 \mathrm{hr}$, respectively. In both cases, the secondary is assumed to be a neutron star with an initial mass of $1.4 M_{\odot}$, and the calculations include angular momentum losses due to gravitational radiation, magnetic braking and mass loss from the system. For comparison, the dot-dashed curve shows the Eddington accretion rate for a neutron star.

and the duration of the LMXB phase may be much shorter than was found in earlier calculations. If there is a sufficiently large population of LMXBs with subgiant secondaries, this could solve the apparent statistical discrepancy between the number of LMXBs and millisecond pulsars.

\section{Caveats and Conclusions}

Our calculations so far have only been exploratory and did not include several effects that may change our conclusions quantitatively as well qualitatively. The most important of these are:

- The effects of aspherical illumination. Since in LMXBs only one side is illuminated, hydrostatic equilibrium necessarily breaks down. This is likely to lead to internal non-radial circulation, which could - in principle - prevent the inflation of the secondary.

- Radiation-induced winds. These will be particularly important near the end and after the semidetached phase.

- Shielding effects (for example due to a flaring disk), which may introduce a negative feedback and may limit the maximum mass-transfer rate.

Despite these caveats, we conclude that, because of the simplicity of the physical process and the large number of potential applications, irradiation effects, as discussed here, are likely to be important and essential for a comprehensive understanding of LMXBs.

\section{Reference}

Podsiadlowski, Ph. (1991), Nature 350, 654. 